\title{
In vivo binding of PRDM9 reveals interactions with noncanonical genomic sites
}

\author{
Corinne Grey, ${ }^{1,6}$ Julie A.J. Clément, ${ }^{1,6}$ Jérôme Buard, ${ }^{1}$ Benjamin Leblanc, ${ }^{2}$ Ivo Gut, ${ }^{3,4}$ \\ Marta Gut, ${ }^{3,4}$ Laurent Duret, $^{5}$ and Bernard de Massy ${ }^{1}$ \\ ${ }^{1}$ Institut de Génétique Humaine UMR9002 CNRS-Université de Montpellier, 34396 Montpellier Cedex 05, France; ${ }^{2}$ Biotech Research \\ and Innovation Centre (BRIC), University of Copenhagen, 2200 Copenhagen, Denmark; ${ }^{3}$ CNAG-CRG, Centre for Genomic Regulation \\ (CRG), Barcelona Institute of Science and Technology (BIST), 08028 Barcelona, Spain; ${ }^{4}$ Universitat Pompeu Fabra (UPF), 08003 \\ Barcelona, Spain; ${ }^{5}$ Université de Lyon, Université Claude Bernard, CNRS, Laboratoire de Biométrie et Biologie Evolutive UMR 5558, \\ F-69100, Villeurbanne, France
}

\begin{abstract}
In mouse and human meiosis, DNA double-strand breaks (DSBs) initiate homologous recombination and occur at specific sites called hotspots. The localization of these sites is determined by the sequence-specific DNA binding domain of the PRDM9 histone methyl transferase. Here, we performed an extensive analysis of PRDM9 binding in mouse spermatocytes. Unexpectedly, we identified a noncanonical recruitment of PRDM9 to sites that lack recombination activity and the PRDM9 binding consensus motif. These sites include gene promoters, where PRDM9 is recruited in a DSB-dependent manner. Another subset reveals DSB-independent interactions between PRDM9 and genomic sites, such as the binding sites for the insulator protein CTCF. We propose that these DSB-independent sites result from interactions between hotspot-bound PRDM9 and genomic sequences located on the chromosome axis.
\end{abstract}

[Supplemental material is available for this article.]

Recombination between homologous chromosomes is required for proper chromosome segregation at the first meiotic division in the majority of sexually reproducing organisms. This specific recombination pathway is initiated by the formation of DNA double-strand breaks (DSBs) (de Massy 2013) that are generated by the SPO11/TOPOVIBL protein complex (Robert et al. 2016; Vrielynck et al. 2016) and repaired by homologous recombination (Baudat et al. 2013; Hunter 2015). DSB repair leads to reciprocal and nonreciprocal exchanges of genetic material between paternal and maternal chromosomes, called cross-overs and gene conversions, respectively. Meiotic recombination is essential for fertility in most species and is a major source of genome diversity (Chen et al. 2007; Coop and Przeworski 2007).

Meiotic recombination takes place during extensive chromosome reorganization at meiotic prophase. Chromosomes arrange as an array of chromatin loops that are anchored to a protein axis, made of cohesins and other structural proteins. This structure serves as a platform for various members of the recombination machinery and for regulating the recombination activity (Zickler and Kleckner 1999). This loop-axis configuration plays an important role in the regulation of DSB formation via induction (Borde and de Massy 2013) and inhibition (Thacker et al. 2014) of DSB activity.

In Saccharomyces cerevisiae, where DSB sites have been analyzed in detail, DSBs preferentially occur in loops and in accessible nucleosome-depleted chromatin regions of $\sim 200 \mathrm{bp}$, which are called DSB hotspots and which are mainly located within promot-

\footnotetext{
${ }^{6}$ These authors are co-first authors and contributed equally to this work.

Corresponding authors: corinne.grey@igh.cnrs.fr; bernard.demassy@igh.cnrs.fr

Article published online before print. Article, supplemental material, and publication date are at http://www.genome.org/cgi/doi/10.1101/gr.217240.116. Freely available online through the Genome Research Open Access option.
}

er regions, upstream of transcription start sites. Hotspots are flanked by positioned nucleosomes that are enriched in trimethylation of lysine 4 of histone H3 (H3K4me3) (Pan et al. 2011). This histone modification is deposited by SET1, a subunit of the COMPASS complex recruited by RNA polymerase II. Although not absolutely required, H3K4me3 is important for normal DSB levels and localization (Borde et al. 2009). SPP1, a member of the SET1 complex binds to H3K4me3 and to MER2, an essential member of the meiotic DSB formation machinery (Acquaviva et al. 2013; Sommermeyer et al. 2013), located on the axis of meiotic chromosomes (Panizza et al. 2011). The SPP1-mediated interactions are thought to tether and/or stabilize the hotspot region to the axis where DSBs are predicted to occur (Blat et al. 2002; Panizza et al. 2011).

In mice and humans, meiotic recombination hotspots are determined by the DNA sequence specificity of the PRDM9 zinc finger domain (Baudat et al. 2010; Myers et al. 2010; Parvanov et al. 2010). Different from $S$. cerevisiae, these hotspots are not preferentially located in promoter regions, but they are enriched in H3K4me3 (Buard et al. 2009; Smagulova et al. 2011), presumably through PRDM9 methyltransferase activity (Hayashi et al. 2005). Indeed, PRDM9 can methylate histone $\mathrm{H} 3$ at $\mathrm{K} 4, \mathrm{~K} 9$, and $\mathrm{K} 36$ in vitro (Wu et al. 2013; Eram et al. 2014; Koh-Stenta et al. 2014). Like H3K4me3, H3K36me3 also can be associated with nucleosomes adjacent to hotspots (Buard et al. 2009; Davies et al. 2016; Powers et al. 2016). Hotspot-associated H3K4me3 is detected as early as $9 \mathrm{~d}$ post-partum (dpp) in mouse testis when spermatocytes begin to enter leptonema, the first stage of meiotic prophase (Grey et al. 2011). This H3K4me3 enrichment does not depend on Spo11, consistent with a role before DSB formation (Buard et al. 2009; Grey et al. 2011; Brick et al. 2012; Borde and de Massy 2013).

(c) 2017 Grey et al. This article, published in Genome Research, is available under a Creative Commons License (Attribution 4.0 International), as described at http://creativecommons.org/licenses/by/4.0/. 
However, it has been hypothesized that H3K36me3 is involved in DSB repair by homologous recombination in mammalian cells (Aymard et al. 2014; Carvalho et al. 2014; Pfister et al. 2014), thus raising the question of when and how this mark is deposited and whether its deposition depends on DSB formation.

A remarkable feature of PRDM9 is its $\mathrm{C} 2 \mathrm{H} 2$ zinc finger domain that enables the protein to recognize specific DNA motifs and to tether initiation of meiotic recombination to specific sites in the genome. This zinc finger domain, which has a minisatellite-like structure, is highly polymorphic in mice and humans (Berg et al. 2010; Buard et al. 2014; Kono et al. 2014) and mutates at a high rate in the human germ line (Jeffreys et al. 2013). Prdm9 zinc finger alleles differ mostly by the number of zinc fingers or by substitutions of amino acids involved in the interaction with DNA, thus leading to variability in the DNA sequence specificity. In mice and humans, it has been directly demonstrated that meiotic DSBs are specified by PRDM9 by chromatin immunoprecipitation and sequencing (ChIP-seq) of the binding sites of the DMC1 strand invasion protein. DMC1 binds to single-strand DNA generated by DSB end processing (Smagulova et al. 2011). By use of this approach, it was shown, in mice and humans, that PRDM9 variants with different zinc finger arrays specify distinct, essentially not overlapping, sets of meiotic DSB sites throughout the genome (Brick et al. 2012; Pratto et al. 2014; Smagulova et al. 2016). PRDM9, which belongs to the PRDM family of transcription regulators (Fog et al. 2012), has homologs in most mammals with the exception of Canidae. However, PRDM9 is absent, is not functional, or is not clearly identifiable in all other vertebrate lineages examined to date. In dogs and birds, where PRDM9 is nonfunctional or absent, meiotic hotspots preferentially localize to functional genomic elements that are enriched in H3K4me3, such as transcription start sites and/or CpG islands, a chromatin environment sharing similarities with $S$. cerevisiae DSB sites (MunozFuentes et al. 2011; Axelsson et al. 2012; Auton et al. 2013; Singhal et al. 2015). Strikingly, when $\operatorname{Prdm} 9$ is inactivated in mice, DSB formation still occurs, but at new locations that mainly correspond to promoter regions, which are also enriched in H3K4me3 (Brick et al. 2012). However, for unknown reasons, the downstream repair pathway is partially defective and meiotic progression is altered. Thus, in the mouse, PRDM9 is indispensable for normal fertility (Hayashi et al. 2005).

Here, we performed PRDM9 ChIP-seq using chromatin from mouse testes to analyze PRDM9 binding sites and to evaluate the relationship between PRDM9 sites and meiotic DSB formation. For this, we used two mouse strains with different Prdm9 alleles with distinct zinc finger domains. We mapped H3K4me3, H3K36me3, and DMC1 distribution by ChIP-seq in the same strains. We also investigated the functional relationship between DSB formation, PRDM9 binding, and H3K36me3 enrichment by analyzing PRDM9 binding and H3K36me3 enrichment in Spo11deficient mice.

\section{Results}

\section{Identification of two classes of PRDM9 binding sites}

To analyze PRDM9 binding to chromatin in optimal conditions, we purified chromatin from testes of prepuberal mice at $13 \mathrm{dpp}$, where the relative abundance of early stages of meiotic prophase is elevated. We used two mouse strains (C57BL/6, hereafter B6; and RJ2) that harbor Prdm9 alleles with distinct zinc finger arrays that specify distinct recombination hotspot localizations (Baudat et al. 2010; Grey et al. 2011). The allele present in B6 mice is from Mus musculus domesticus (named Prdm $9^{\text {Dom } 2}$ ) and the allele present in RJ2 mice is from Mus musculus castaneus (named $\operatorname{Prdm} 9^{\mathrm{Cst}}$ ) (Fig. 1A). The genetic background of these two strains is $\mathrm{C} 57 \mathrm{BL} / 6$ and C57BL/10, respectively, two nearly identical inbred strains derived from C57BL at the beginning of the 20th century. The anti-PRDM9 antibody used detected a nuclear signal in B6 spermatocytes at leptonema and zygonema that was absent in Prdm $9^{-/-}$B6 spermatocytes (B6 Prdm9 ${ }^{K O}$ hereafter) (Supplemental Figs. S1A, S1B). This antibody recognized efficiently both PRDM9 ${ }^{\text {Dom2 }}$ and PRDM9 ${ }^{\text {Cst }}$ variants (Supplemental Fig. S1C).

ChIP-seq experiments revealed 3041 and 10,871 PRDM9 binding sites in the B6 and RJ2 strains, respectively (Fig. 1B). The reproducibility between replicates was tested and taken into account using the irreproducible discovery rate (IDR) method (Supplemental Table S1; Supplemental Material). Their specificity was validated by the analysis of $\mathrm{B} 6 \operatorname{Prdm} 9^{K O}$ mice, using nonstringent condition (a relaxed $P$-value) for peak calling, allowing us to remove nonspecific signals (see Supplemental Material). In addition, the few peaks shared between strains (213 peaks), which could be nonspecific, were removed from this pool and analyzed separately, leading to 2601 and 10,121 PRDM9 binding regions specific for the B6 and RJ2 strains, respectively (Fig. 1B). The striking difference in peak numbers between the two strains was not due to a difference in progression in meiosis (Supplemental Fig. S1D). We speculate that the lower number and lower average strength (Supplemental Fig. S2A) of PRDM9 $9^{\text {Dom2 }}$ peaks compared with PRDM9 ${ }^{\text {Cst }}$ is biologically relevant. This could be due to an overall lower affinity of PRDM9 ${ }^{\text {Dom2 }}$ for its binding sites, leading to a reduced occupancy, as detected by ChIP analysis.

PRDM9 binding to DNA is linked to at least two detectable molecular events: H3K4me3 enrichment and DSB formation, which can be monitored by DMC1 association with DSB ends. H3K4me3 and DMC1 profiles have been previously monitored in strains closely related to B6 that express PRDM9 ${ }^{\text {Dom2 }}$ (Smagulova et al. 2011; Brick et al. 2012), as well as the H3K4me3 profile in a mouse strain that expresses a genetically engineered $\operatorname{Prdm} 9^{\mathrm{Cst}}$ allele in the C57BL/6 background (Baker et al. 2014). Therefore, we mapped DMC1 and H3K4me3 in the B6 and RJ2 strains by ChIP-seq experiments (Supplemental Table S1). We identified about 15,000 DMC1 peaks in each strain that overlap by $<1 \%$ (14,774 for B6 and 15,195 for RJ2) (Fig. 1C). The similar number of DMC1 peaks in the two strains is in contrast with the lower number and reduced average intensity of PRDM9 peaks in B6 compared with RJ2 testes. H3K4me3 enrichments were quantitatively and qualitatively similar to those reported in previous studies. Specifically, most H3K4me3 sites were shared by both B6 and RJ2 strains (79\% of total B6 peaks and $63 \%$ of total RJ2 peaks) (Fig. 1C) and significantly overlapped with regulatory elements in the genome ( $60 \%$ of common H3K4me3 peaks overlapped with annotated transcription start sites or testes-specific enhancers). Strain-specific H3K4me3 peaks $(10,835$ for B6 and 24,185 for RJ2) mirrored the site-specific PRDM9 methyltransferase activity, as previously reported (Brick et al. 2012; Baker et al. 2014). The difference in the number of H3K4me3 and DMC1 peaks, compared with PRDM9, could be due to various reasons, for instance, the ChIP assay sensitivity or the shorter half-life of PRDM9 association with its binding sites compared with that of DMC1 or H3K4me3.

Unexpectedly, the comparative analysis of the PRDM9, DMC1, and H3K4me3 sites revealed two strikingly different types 
A

B6 (PRDM9 $9^{\text {Dom2 }) ~ * ~}$

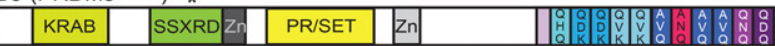

RJ2 (PRDM9 ${ }^{\mathrm{Cst}}$ )

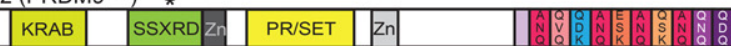

C

\begin{tabular}{|l||c|c|c|}
\hline Number of peaks & B6 (PRDM9 & \\
\hline \hline PRDMm $)$ & RJ2 (PRDM9 $^{\text {cst }}$ ) & Common \\
\hline DMC1 & 2,601 & 10,121 & 213 \\
\hline H3K4me3 & 14,643 & 15,064 & 131 \\
\hline
\end{tabular}

B

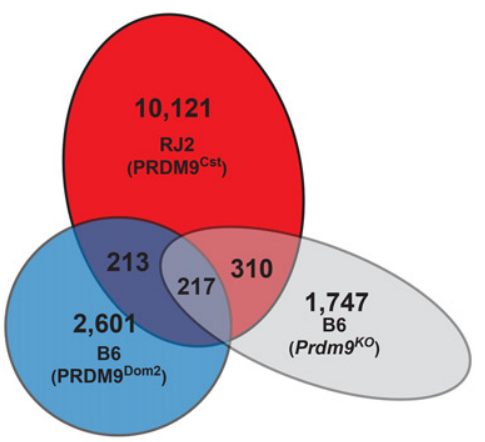

D

\begin{tabular}{|l||c|c|c|c|c|c|}
\hline B6 (PRDM9 ${ }^{\text {Dom2 }}$ ) & a & b & c & d & A & B \\
\hline \hline PRDM9 & + & + & + & + & + & + \\
\hline DMC1 & + & + & + & - & - & - \\
\hline H3K4me3 (B6) & + & $?$ & - & + & $?$ & - \\
\hline H3K4me3 (both) & - & + & - & - & + & - \\
\hline Number of peaks & 1802 & 38 & 17 & 39 & 459 & 246 \\
\hline & & & & & & \\
\hline RJ2 (PRDM9 ${ }^{\text {cst) }}$ & a & b & c & d & A & B \\
\hline \hline PRDM9 & + & + & + & + & + & + \\
\hline DMC1 & + & + & + & - & - & - \\
\hline H3K4me3 (RJ2) & + & $?$ & - & + & $?$ & - \\
\hline H3K4me3 (both) & - & + & - & - & + & - \\
\hline Number of peaks & 6695 & 107 & 28 & 527 & 425 & 2239 \\
\hline
\end{tabular}

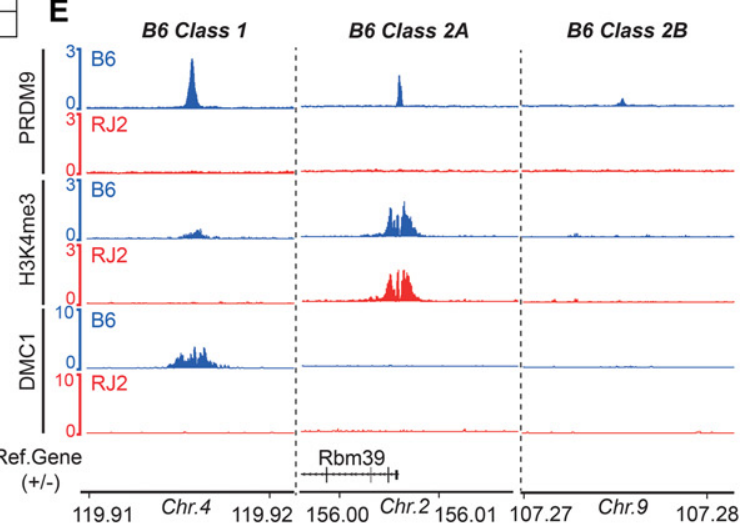

$\mathbf{F}$
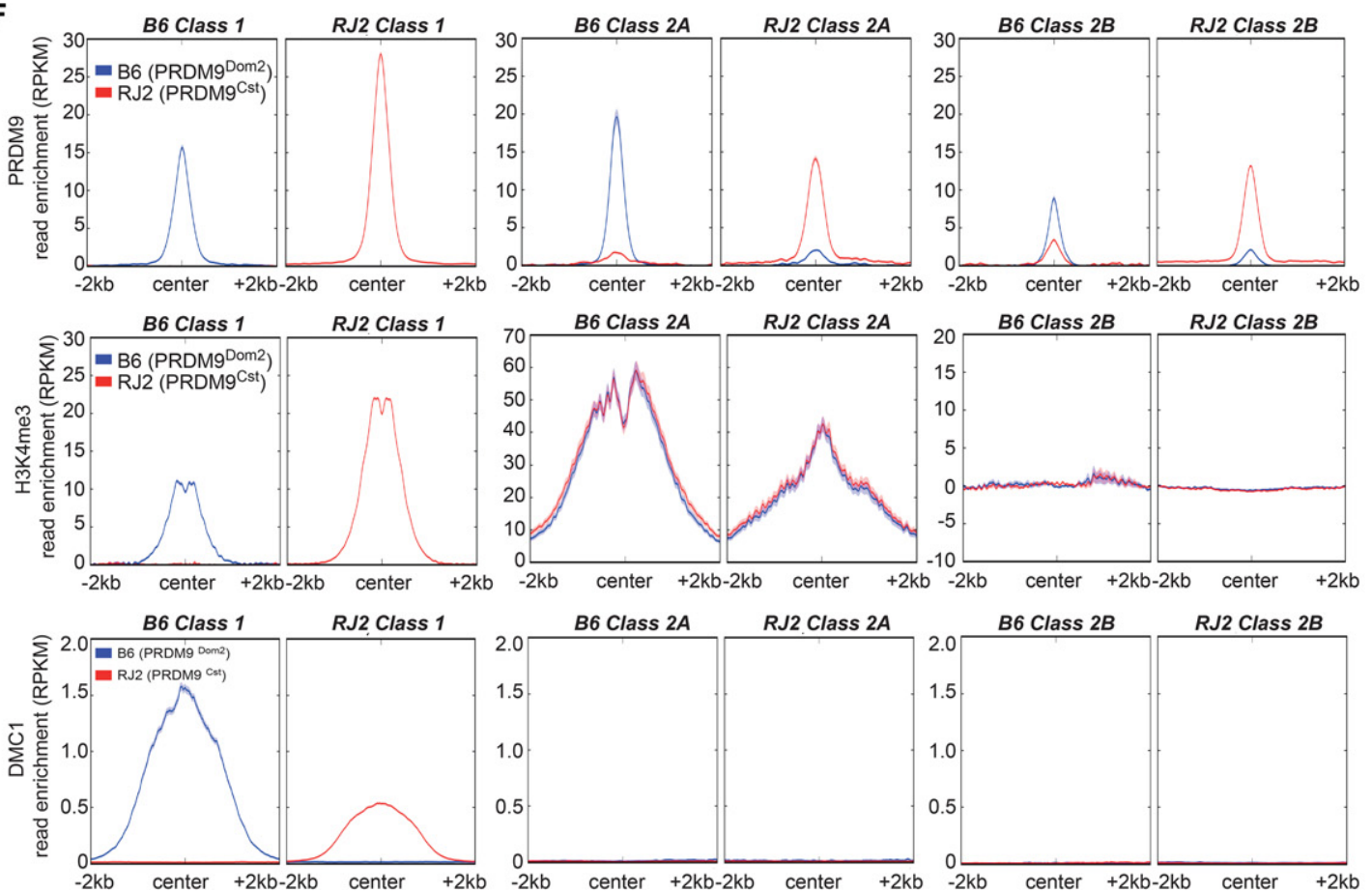

Figure 1. PRDM 9 binds to two distinct classes of sites in the genome. $(A)$ Schematic representation of the PRDM9 $9^{\text {Dom2 }}$ and PRDM9 ${ }^{\text {Cst }}$ proteins in the B6 and R/2 mouse strains, respectively. The asterisk indicates the only amino acid substitution outside the zinc finger array due to a nonsynonymous singlenucleotide polymorphism. (B) Venn diagram showing the overlap of PRDM9 ChIP-sequencing peaks in B6, RJ2, and B6 Prdm $9^{K O}$ mice. (C) Number of strain specific and common peaks retrieved for PRDM9, DMC1, and H3K4me3 ChIP-seq experiments. (D) Classification of PRDM9 peaks in subclasses with (+) or without (-) enrichment for DMC1 and/or H3K4me3 in B6 and RJ2 mice. Question marks indicate situations where a PRDM9-dependent signal (if present) could not be detected because of a common signal also found in the other strain. Class 1 contains PRDM9 peaks that are enriched in DMC1 and/or strainspecific (PRDM9-dependent) H3K4me3. Class 2 sites are negative for DMC1 but can overlap peaks that are enriched for H3K4me3 in both mouse strains. (E) Read distribution from PRDM9, H3K4me3, and DMC1 ChIP-seq experiments at representative B6 class 1, 2A, and 2B sites in B6 and RJ2 mice. Read distribution was calculated from pooled replicates, in 1-bp windows, and normalized by library size and input. $(F)$ Average read enrichment (reads per kilobase pair per million mapped reads [RPKM]) of PRDM9, H3K4me3, and DMC1 in B6 and RJ2 mice centered on class 1, 2A, and 2B sites in each strain.

\section{Genome Research}

www.genome.org 
of PRDM9 binding sites in both strains. Most binding sites showed the properties expected for PRDM9's role in specifying DSB formation, namely, enrichment for H3K4me3 and DMC1 in addition to PRDM9. We called these sites class 1 sites (73\% for both $\mathrm{B} 6$ and RJ2). The other binding sites (class 2 sites) showed no detectable DMC1 signal and were enriched (class 2A) or not (class 2B) in PRDM9-independent H3K4me3 (Fig. 1D-F; Supplemental Fig. S2B). Class $2 \mathrm{~B}$ peaks were on average of lower strength compared with that of class 1 peaks (Supplemental Fig. S2C).

Among class 1 peaks, the average strength of DMC1 was greater in B6 compared with RJ2 (Supplemental Fig. S2D) despite the greater strength of PRDM9 peaks from RJ2 mentioned above (Supplemental Fig. S2A). Among all DMC1 sites, class 1 peaks correspond to the strongest intensity sites, in agreement with our hypothesis that PRDM9 detection is less sensitive than DMC1 detection in our assays (Supplemental Fig. S2E). In class 1 peaks, the average H3K4me3 level was higher in RJ2 than in B6 samples (Fig. 1F), in agreement with the stronger PRDM9 signal observed in RJ2 samples (Fig. 1F; Supplemental Fig. S2A). Overall, we detected a slightly higher correlation between PRDM9 and H3K4me3 than between PRDM9 and DMC1 in both the B6 and RJ2 samples (Supplemental Fig. S2F).

In most class 1 peaks, H3K4me3 enrichment was strain specific, as expected if this histone modification was catalyzed by PRDM9 (Fig. 1D). A small percentage of class 1 peaks (class 1b; $2 \%$ for both B6 and RJ2) showed H3K4me3 enrichment in both strains. This could be explained by the presence of an overlapping gene regulatory element $(79 \%$ and $65 \%$ of these class $1 \mathrm{~b}$ peaks for B6 and for RJ2, respectively, overlapped with a putative promoter or enhancer). This overlap did not allow assessing the presence of PRDM9-dependent H3K4me3 enrichment in these regions. A small fraction of class 1 (class $1 \mathrm{~d}$ : $2 \%$ in $\mathrm{B} 6,7 \%$ in RJ2) peaks did overlap with strain-specific H3K4me3, but not with DMC1. Nevertheless, these sites showed signs of recombination activity (see below).

\section{PRDM9 binding sites without recombination activity}

Unexpectedly, class 2 PRDM9 binding sites did not show any detectable DMC1 enrichment (Fig. 1E,F; Supplemental Fig. S2B). Therefore, these sites should not correspond to recombination sites, although a faster DMC1 turnover at these sites cannot be excluded. We thus performed an alternative analysis based on GC content evolution, which is entirely independent from technical and molecular issues. Meiotic recombination activity generates GC-biased gene conversion (Duret and Galtier 2009), and this bias leads to increased rates of A:T-to-G:C substitutions. This effect has been detected at mouse recombination hotspots, defined by DMC1 ChIP-seq (Clement and Arndt 2013). GC-biased gene conversion can be measured by estimating the GC content at equilibrium, also named GC* (see Methods). At class 1 sites, a sharp GC* increase was detected at the center of hotspots, defined by PRDM9 binding, specifically in the lineage where the corresponding PRDM9 allele was present (M. m. domesticus for PRDM9 ${ }^{\text {Dom2 }}$, M. m. castaneus for PRDM9 ${ }^{\mathrm{Cst}}$ ) (Fig. 2A). As expected, GC* level was correlated with PRDM9 peak strength (Supplemental Fig. S3A). Conversely, at class 2 sites (and also classes $2 \mathrm{~A}$ and 2B separately) (Supplemental Fig. S3B), no GC* increase was observed, indicating the lack of detectable historical recombination activity in these genomic regions (Fig. 2B). The absence of GC* increase in class 2 sites was not due to a limited sample size, because a random subsampling of the same number of class 1 peaks still allowed the
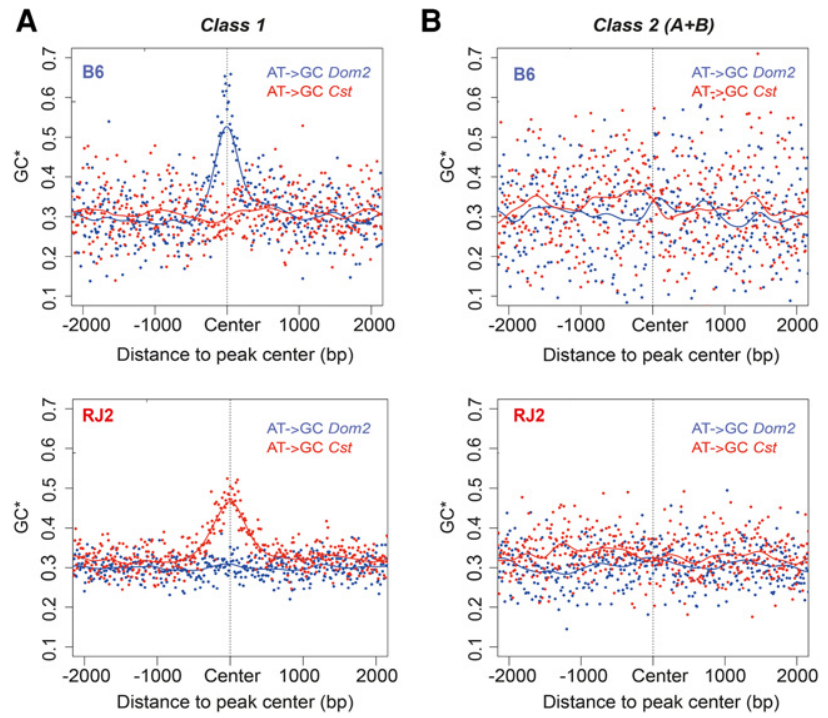

Figure 2. PRDM9 class 1, but not class 2, sites show signatures of GC-biased gene conversion. Points represent the equilibrium GC-content (GC*) estimated from the lineage-specific substitutions aggregated in 10-bp bins from the center of all peaks. GC* in M. m. domesticus and M. m. castaneus lineages are displayed in blue and red, respectively. Lines were obtained by fitting a cubic smoothing spline. $(A) \mathrm{GC}^{*}$ centered on PRDM9 class 1 sites in $\mathrm{B} 6$ and RJ2 mice. $(B) \mathrm{GC}^{*}$ centered on PRDM9 class 2 sites in B6 and RJ2 mice; the equilibrium GC-content $\left(\mathrm{GC}^{*}\right)$ in $M$. $m$. domesticus and $M$. $m$. castaneus lineages are not significantly different (Student $P$-value $=0.65$ and 0.99 for $\mathrm{B} 6$ and $\mathrm{RJ} 2$, respectively).

detection of GC conversion bias (Supplemental Fig. S3C). The subset of class 1d sites in RJ2 samples (527 sites), although devoid of DMC1 enrichment, also showed statistically significant biased GC gene conversion, indicating that these sites are recombination initiation sites (Supplemental Fig. S3D).

At class 2A sites, H3K4me3 level did not correlate with that of PRDM9, suggesting that this enrichment was catalyzed by other methyltransferases, such as those involved in gene expression regulation (Supplemental Fig. S2G). At these sites, the average H3K4me3 enrichment was higher than that of strain-specific H3K4me3 (Fig. 1F). We thus could not determine whether low PRDM9 methyltransferase activity was present at these class 2A sites.

\section{H3K36me3 is specifically enriched at recombination sites in a Spoll-independent manner}

PRDM9 catalyzes H3K36me3 formation in vitro (Wu et al. 2013; Eram et al. 2014), and H3K36me3 enrichment is detected in vivo at the center of mouse hotspots (Davies et al. 2016; Powers et al. 2016). Interestingly, we only detected this PRDM9-dependent H3K36me3 enrichment in class 1 PRDM9 binding sites and not in classes $2 \mathrm{~A}$ or $2 \mathrm{~B}$ (Fig. 3).

As class 1 sites are also recombination sites, it was important to determine whether H3K36me3 enrichment was dependent on recombination activity. To this aim, we monitored H3K36me3 distribution on chromatin from B6 Spo11 $1^{\mathrm{tm} 1 \mathrm{M}}$ (B6 Spo11 ${ }^{K O}$ hereafter) testes, where initiation of recombination activity is abolished. H3K36me3 level at PRDM9 ${ }^{\text {Dom2 }}$ sites was similar in B6 Spo11 ${ }^{\text {KO }}$ and B6 samples (Fig. 3), demonstrating that this modification does not rely on SPO11-dependent DSBs. Therefore, H3K36me3 

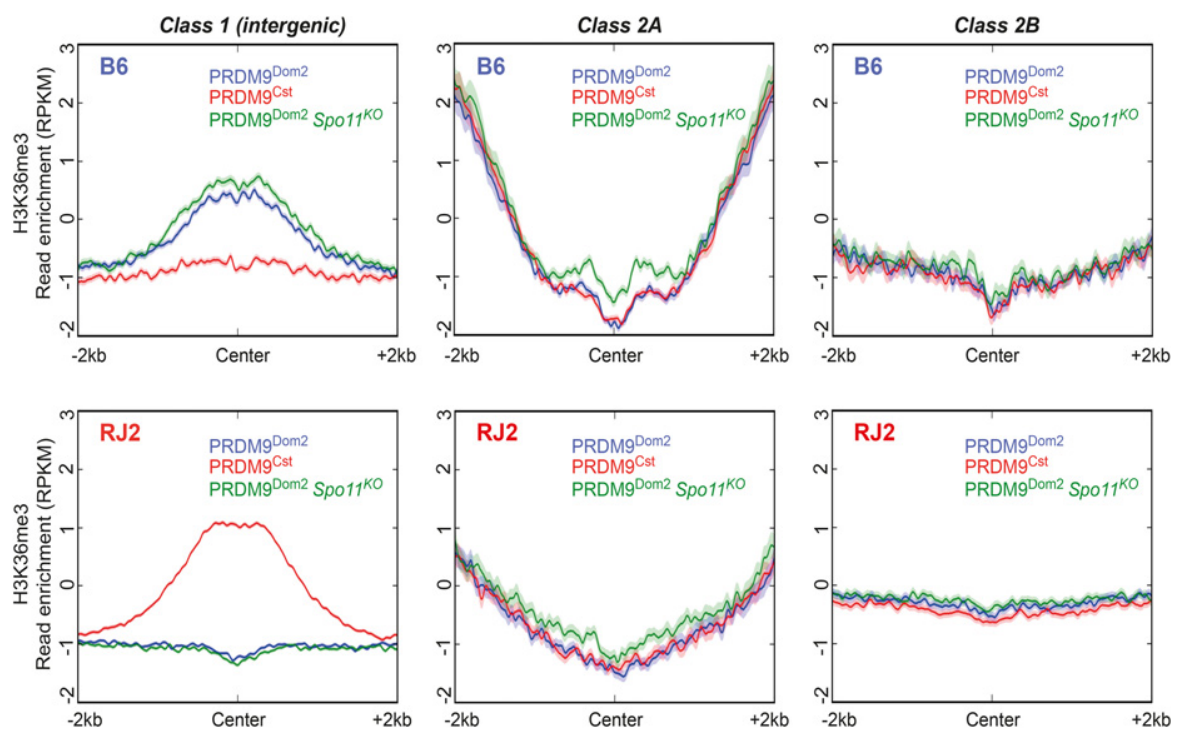

Figure 3. PRDM9-dependent $\mathrm{H} 3 \mathrm{~K} 36 \mathrm{me} 3$ enrichment in class 1 sites is independent of double-strand break formation. Average and normalized read enrichment (RPKM) of H3K36me3 in B6 (blue), B6 $\mathrm{Spo}_{1}{ }^{\mathrm{KO}}$ (green), and RJ2 (red) mice. B6 and B6 Spo11 ${ }^{\mathrm{KO}}$ mice have the same PRDM9 allele (Dom2). Read enrichments are centered on PRDM9 class 1, 2A, and 2B sites and normalized by subtracting the input read enrichment. Only peaks that overlapped with intergenic regions were considered for class 1 (826 and 3321 sites for B6 and RJ2, respectively) to avoid noise from the H3K36me3 signal specifically found at transcribed genes.

at recombination sites could be directly catalyzed by PRDM9 methyltransferase activity.

\section{Epigenetic features of non-DSB sites bound by PRDM9}

Class 2 sites were not enriched for H3K36me3 around the PRDM9 binding sites, which is compatible with the absence of PRDM9 methyltransferase activity. However, class $2 \mathrm{~A}$ sites, which are highly enriched in H3K4me3, were enriched in H3K36me3 in the flanking regions outside the center of the PRDM9 peaks (Fig. 3). This might reflect an overlap of PRDM9 binding sites with promoters and the transcriptional activity of the associated genes. Indeed, in $\mathrm{B} 6$ and RJ2 testes, $88 \%$ and $72 \%$ of class $2 \mathrm{~A}$ sites, respectively, overlapped with promoters (Supplemental Fig. S4A). To assess histone mark enrichment in class $1,2 \mathrm{~A}$, and $2 \mathrm{~B}$ sites, we took advantage of available ChIP-seq data for several histone marks in $\mathrm{B} 6$ mice: H3K27me3, H3K27ac, and H3K4me1 in spermatocytes from Hammoud et al. (2014); lysine crotonylation (Kcr) in spermatocytes from Tan et al. (2011); and RNA polymerase II (Pol2) in whole testis (Mouse ENCODE Project) (Yue et al. 2014). Class 2A sites from B6 mice and also from RJ2 mice, although to a lesser extent, were strongly enriched in marks associated with active promoters (Supplemental Fig. S4B). The enrichment was centered on the PRDM9 peak. However, class 2B sites were devoid of any significant epigenetic feature. Moreover, genomic features (promoter, genic, and intergenic regions) were stochastically distributed within this class (Supplemental Fig. S4A).

Consistent with the epigenetic marks observed at class $2 \mathrm{~A}$ sites, these sites were characterized by high CpG density, and most of them overlapped with CpG islands (85\% and 53\% in B6 and RJ2, respectively). Conversely, class 1 and class $2 \mathrm{~B}$ sites rarely overlapped with CpG islands ( $\sim 1 \%$ and $\sim 6 \%$, respectively). We examined the density of class $2 \mathrm{~A}$ sites per chromosome and detected a strong positive correlation with $\mathrm{CpG}$ islands (Supplemental Fig. S4C). Class 2B sites showed similar patterns, whereas the density in class 1 sites was largely independent of the CpG island content, at the resolution of $1 \mathrm{Mb}$ (Supplemental Fig. S4C). We analyzed the distribution of sites along chromosomes within 10$\mathrm{Mb}$ windows and detected a nonstochastic distribution of PRDM9 ${ }^{\text {Cst }}$ class 1 sites as shown for Chromosome 1 (Supplemental Fig. S4D). Interestingly, the distribution of PRDM9 ${ }^{\text {Cst }}$ class $2 \mathrm{~B}$ sites on Chromosome 1 was similar, and the genome-wide distribution of class 1 sites correlated with that of class $2 \mathrm{~B}$ sites, reaching a correlation coefficient of 0.71 for $10-\mathrm{Mb}$ window size (Supplemental Fig. S4D). The correlation between PRDM9 $^{\text {Cst }}$ class 1 and class $2 \mathrm{~A}$ was weaker, as well as the one observed for the analysis of B6 sites likely due to small sample size (Supplemental Fig. S4D).

\section{PRDM9 is recruited to non-DSB sites that do not contain its DNA binding motif}

At mouse DMC1 binding sites, consensus motifs that partially overlap with the in silico prediction of the zinc finger domain specificity for the $\operatorname{Prdm} 9^{\text {Dom } 2}$ allele have been identified (Smagulova et al. 2011; Brick et al. 2012). Similarly, PRDM9 ${ }^{\text {Cst }}$-specific H3K4me3 sites overlap with a consensus motif for the $\operatorname{Prdm} 9^{\text {Cst }}$ allele (Baker et al. 2014). Therefore, we asked whether consensus motifs were present in each PRDM9 binding site class. We identified a motif that matched the previously identified motifs (Supplemental Fig. S5A,B) in both PRDM9 ${ }^{\text {Dom2 }}$ and PRDM99 ${ }^{\text {Cst }}$ class 1 sites, but not in class 2 peak DNA sequences. We then investigated the presence of PRDM9 class 1 consensus motifs in all identified peaks and found that their position overlapped with the center of the PRDM9 peak in class 1 sites (Fig. 4). Conversely, we did not find any significant enrichment for class 1 consensus PRDM9 ${ }^{\text {Dom2 }}$ or PRDM9 ${ }^{\mathrm{Cst}}$ motifs in class $2 \mathrm{~A}$ and $2 \mathrm{~B}$ peaks (Fig. 4). This method was sensitive enough to also detect the presence of PRDM9 $9^{\text {Dom2 }}$ or PRDM9 ${ }^{\text {Cst }}$ motifs in class $1 \mathrm{~d}$ peaks where the DMC1 signal was undetectable (Supplemental Fig. S5C). These observations strongly suggest that PRDM9 does not bind to class 2 sites through its zinc finger domain or at least not via the zinc fingers involved in recombination site specification. As class 2 A sites overlap heavily with promoters, one may speculate that PRDM9 is recruited to these sites by interacting with some transcription machinery components.

PRDM9 ${ }^{\text {Dom2 }}$ class $2 \mathrm{~B}$ sites were enriched in a motif similar to the one recognized by the insulator protein CTCF (Supplemental Fig. S6A; Nakahashi et al. 2013; Pugacheva et al. 2015). Consistent with this observation, we detected enrichment for the CTCF consensus motif at the center of B6 class 2B sites (Supplemental Fig. S6B). The absolute number of CTCF motif-containing peaks was similar in B6 and RJ2 samples (148 and 259 in B6 and RJ2, respectively, compared with 25 and 38 in randomized controls); however, these sites represented $60 \%$ of all class $2 \mathrm{~B}$ sites in $\mathrm{B} 6$ and only $11 \%$ in RJ2. By coimmunoprecipitation analysis, we

\section{Genome Research}

www.genome.org 


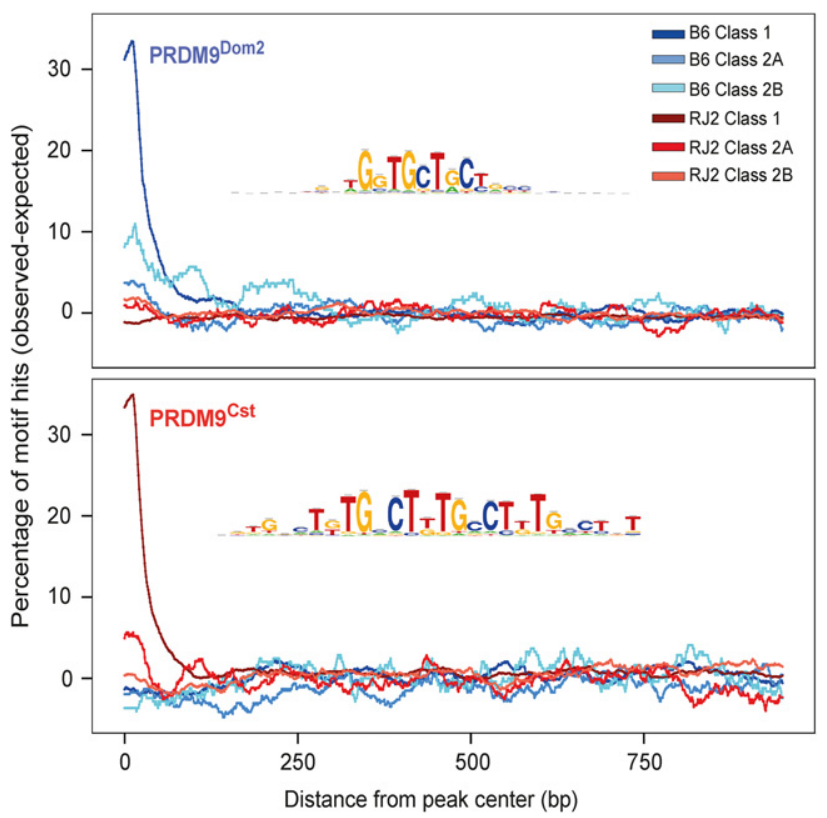

Figure 4. PRDM9 class 1 but not class 2 sites are enriched in PRDM9 allele-specific motifs. Distribution of hits for PRDM $9^{\mathrm{Dom} 2}$ and PRDM9 ${ }^{\mathrm{Cst}}$ motifs (each consensus motif is depicted on each graph) along B6 and RJ2 class 1, 2A, and 2B sites from the center of the PRDM9 sites and up to 1 $\mathrm{kb}$ of distance. Hits were calculated in a 50-bp sliding window with a 1bp step.

revealed that in vivo, PRDM9 interacts with CTCF in testes. This interaction required the presence of DNA and/or RNA because it was lost upon nuclease treatment (Supplemental Fig. S6C). It seems unlikely that PRDM9 interacts directly with a CTCF binding motif because, using in vitro affinity assays, we did not detect any interaction between PRDM9 zinc fingers and a CTCF consensus motif (F Baudat, pers. comm.). Thus, the interaction between PRDM9 and sites containing the CTCF DNA motif could be the result of an indirect interaction between PRDM9 and CTCF. This interaction could involve soluble PRDM9 or PRDM9 bound to its motif (class 1 sites). Both possibilities are compatible with the recovery of DNA sequences containing CTCF motifs by crosslink ChIP. In RJ2, class $2 \mathrm{~B}$ represented $23 \%$ of all sites compared with $9 \%$ in B6. It thus appears that these indirect peaks are recovered more efficiently in RJ2 and that they include, in addition to CTCF sites, a significant number of other genomic sites.

Our analysis also detected a small number of PRDM9 peaks that were common between the two strains B6 and RJ2 (213 peaks) (Fig. 1B; Supplemental Fig. S7A,B) but absent in $\operatorname{Prdm} 9^{K O}$ and that could be sites where PRDM9 binding is not directed by its DNA sequence specificity. Based on DMC1 and H3K4me3 analysis, $27 \%$ and $60 \%$ of these peaks correspond to classes $2 \mathrm{~A}$ and $2 \mathrm{~B}$, respectively (Supplemental Fig. S7C). Motif search showed the absence of motif enrichment for PRDM9 $9^{\text {Dom2 }}$, PRDM9 $9^{\text {Cst }}$, and CTCF (Supplemental Fig. S7D).

PRDM9 binding is Spoll dependent at class 2A, but not at class 1 and class $2 \mathrm{~B}$ sites

SPO11 is responsible for the formation of meiotic DSBs during the leptotene stage in mice (Baudat et al. 2000; Romanienko and Camerini-Otero 2000). On the other hand, PRDM9-dependent H3K4me3/H3K36me3 enrichment at recombination hotspots does not require Spo11 (Fig. 3; Buard et al. 2009). Thus, one could hypothesize that PRDM9 binding to DSB sites occurs prior to DSB formation, independently of Spo11. Conversely, PRDM9 recruitment to all or some of the noncanonical binding sites might depend on DSB formation catalyzed by SPO11. To evaluate the relationship between the different classes of PRDM9 binding sites and recombination initiation, we monitored PRDM9 binding in B6 Spo11 ${ }^{K O}$ testes. We detected 1070 peaks (after filtering out the nonspecific signal that overlapped with $\left.\mathrm{B} 6 \operatorname{Prdm} 9^{K O}\right), 88 \%$ of which $(n=942)$ overlapped with those mapped in B6 (Fig. 5A), and validated by analysis of the two replicates (see Methods). As B6 Spo11 ${ }^{K O}$ peaks overlapped largely with strong intensity B6 peaks, their lower number could reflect a lower sensitivity of this experiment (Fig. 5B). These peaks could be classified into the three classes of 1,2A, and 2B, similarly to the analysis of B6 mice (Fig. 5C-F; Supplemental Fig. S8). The peak distribution in the different classes differed, however, significantly between the B6 and B6 Spo11 ${ }^{K O}$ samples $\left(\chi^{2}=272.26, \mathrm{df}=2, P\right.$-value $\left.<10^{-6}\right)$. Specifically, class $2 \mathrm{~A}$ peaks were almost completely absent in B6 Spo11 ${ }^{K O}$ samples (1010 class 1, 1 class 2A, and 59 class $2 \mathrm{~B}$ sites in B6 Spo11 ${ }^{K O}$, respectively, compared with 1896, 459, and 246 sites in B6) (Figs. 1D, 5F). This difference was not due to the bias toward stronger sites. Indeed, the distribution of the 1070 strongest peaks in $\mathrm{B} 6$ (861 class 1, 198 class 2A, and 11 class 2B sites, respectively) was significantly different compared with that of the B6 Spo11 ${ }^{K O}$ peaks $\left(\chi^{2}=431.24, \mathrm{df}=2, P\right.$-value $\left.<10^{-6}\right)$. We conclude that class $2 \mathrm{~A}$ sites are Spo11 dependent. Conversely, class 2B sites in B6 Spo $11^{K O}$ samples shared the same property as class $2 \mathrm{~B}$ sites in $\mathrm{B} 6$ samples, indicating that these potential indirect interactions between PRDM9 and other genomic sites do not require SPO11.

\section{Discussion}

\section{Direct evidence of PRDM9 binding at recombination hotspots}

We report here the first extensive analysis of the in vivo binding of PRDM9 in two mouse strains that express PRDM9 variants with different zinc finger arrays. We show that PRDM9 binds to two classes of sites: sites at recombination hotspots (class 1) and sites that do not show any sign of recombination (class 2). We also demonstrate that PRDM9 binding to recombination hotspots (class 1 sites) is Spo11 independent.

The detection of a higher number of PRDM9 binding sites and, in average, a greater strength in the strain expressing PRDM9 $^{\text {Cst }}$ compared with the one expressing PRDM9 ${ }^{\text {Dom2 }}$, in a M. m. domesticus genetic background, suggests interesting differences in PRDM9 properties according to the genetic context. It is unlikely that these very substantial differences in binding site number and strength are due to technical artefacts given the many controls included in the experiments. These differences may reflect a lower in vivo occupancy of PRDM $9^{\text {Dom2 }}$ motifs compared with PRDM9 ${ }^{\text {Cst }}$ motifs due to differential affinities of the proteins for their binding sites. However, in vitro binding assays of PRDM9 to DNA sequences from strong hotspots did not reveal any major affinity difference between these zinc finger domains (F Baudat, pers. comm.). This suggests the alternative interpretation that the sites available for PRDM9 binding show, on average, lower affinity for PRDM9 ${ }^{\text {Dom2 }}$ compared to PRDM9 ${ }^{\text {Cst }}$ in the B6 genome. This effect could be caused by erosion of PRDM9 ${ }^{\text {Dom2 }}$ motifs by gene conversion (Myers et al. 2010; Baker et al. 2015). As the PRDM9 ${ }^{\text {Dom } 2}$ protein is produced in the M. m. domesticus C57BL/6 strain and PRDM9 ${ }^{\mathrm{Cst}}$ is a variant derived from M. m. castaneus and 
A
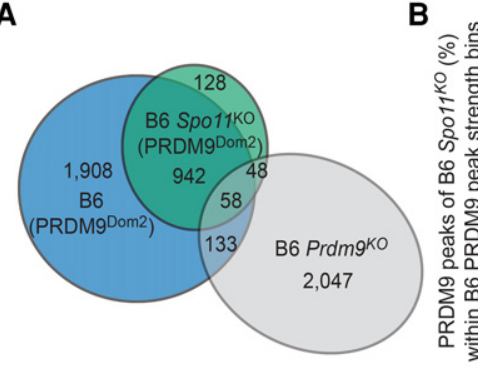

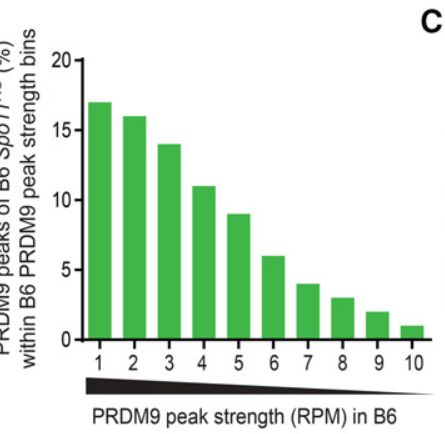

C

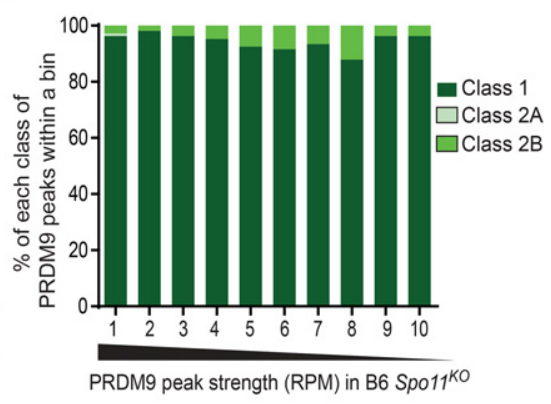

D

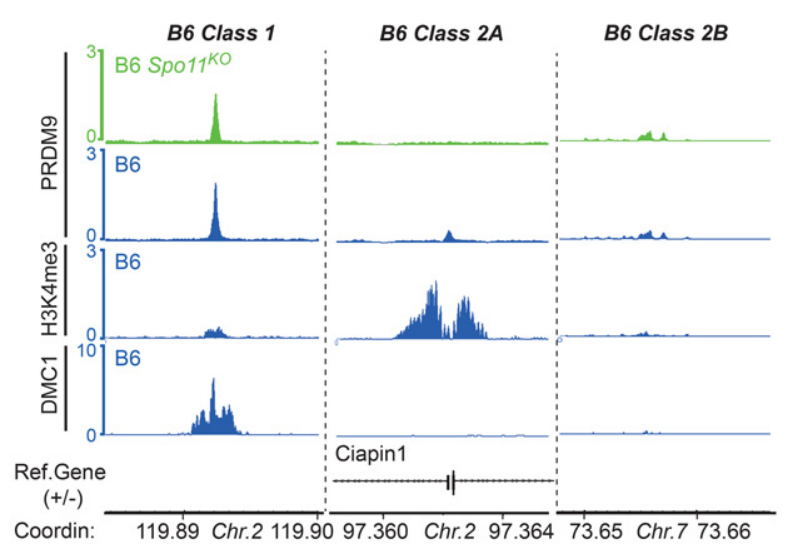

E
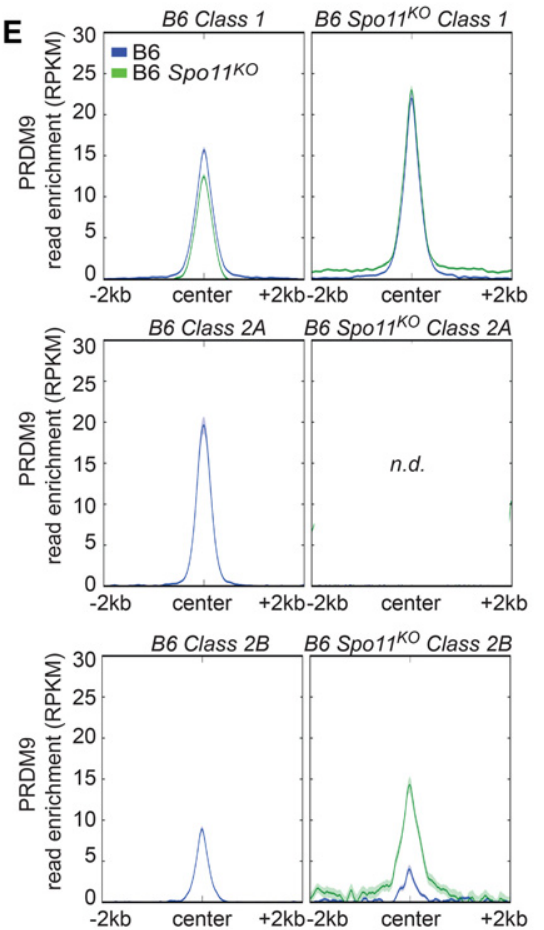

Figure 5. Class $2 \mathrm{~A}$, but not class 1 or class $2 \mathrm{~B}$, sites are Spo11 dependent. ( $A$ ) Venn diagram showing the overlap of PRDM9 ChIP-seq peaks from B6 (all 3041 specific and common peaks), B6 Spo $11^{\mathrm{KO}}$ (PRDM9 ${ }^{\mathrm{Dom} 2}$ ), and B6 Prdm $9^{\mathrm{KO}}$ mice. (B) PRDM9 B6 peaks (Spo $11^{+/+}$) were binned by strength. In each bin, the percentage of B6 Spo1 $11^{\mathrm{KO}}$ (PRDM9 ${ }^{\mathrm{Dom} 2}$ ) overlapping peaks was plotted. (C) Distribution of classes into bins of PRDM9 peak strength monitored in B6 Spo $11^{K O}$ mice. $(D)$ Read distribution from PRDM9 ChIP-seq at representative class 1, 2A, and 2B sites found in B6 Spo $11^{K O}$ mice compared with the read distribution from PRDM9, H3K4me3, and DMC1 ChIP-seq in B6 mice at the same sites. Read distribution was calculated from pooled replicates, in 1-bp windows, and normalized by library size and input. $(E)$ Average read enrichment (RPKM) of PRDM9, in B6 and B6 Spo $11^{\mathrm{KO}}$ mice centered on class $1,2 \mathrm{~A}$, and 2B sites of each strain. (n.d.) Not determined (only one class 2A peak was identified in B6 Spo $11^{\mathrm{KO}}$ ). (F) Classification of the PRDM9-positive peaks detected in B6 Spo $11^{\mathrm{KO}}$ mice in subclasses with (+) or without (-) enrichment for DMC1 (measured in B6 mice) and/or H3K4me3 (specifically present in B6 Spo $11^{\mathrm{KO}}$ mice or also present in RJ2). Classes were defined as in Figure 1D. H3K4me3 ChIP-seq data for B6 Spo 11 ${ }^{\text {KO }}$ (PRDM9 ${ }^{\text {Dom2 }}$ ) are from Brick et al. (2012) and downloaded from the GEO database (GSE35498).

not found in M. m. domesticus (Buard et al. 2014; Kono et al. 2014), erosion should have, indeed, affected only the PRDM9 ${ }^{\text {Dom2 }}$ sites on the $\mathrm{B} 6$ genome present in the analyzed strains.

The level of DMC1 activity (number of sites and reads) was not lower in B6 compared with RJ2 as measured by ChIP-seq. This shows that PRDM9 binding in the B6 strain is not limiting in these conditions and suggests that additional factors that act downstream from PRDM9 binding regulate DSB activity. This is an important feature of DSB regulation that may, in part, explain why hotspots, which were previously identified as sharing the same PRDM9 motif, could have distinct recombination activities (Berg et al. 2011). Mechanistically, one could propose that any step after PRDM9 binding, such as SPO11 recruitment and/or activation, could contribute to this effect.

Histone modifications associated with PRDM9 binding: a role for DSB activation?

The presence of H3K4me3 and H3K36me3 has been detected at nucleosomes around the center of mouse hotspots; H3K4me3, around the center of human hotspots (Fig. 3; Buard et al. 2009; Grey et al. 2011; Smagulova et al. 2011; Pratto et al. 2014; Davies et al. 2016; Powers et al. 2016). These studies showed that these modifications are PRDM9 dependent and thus are predicted to

\section{Genome Research}

www.genome.org 
be catalyzed by PRDM9. Our direct evidence of PRDM9 binding at these sites further supports this hypothesis. Also, as previously shown for $\mathrm{H} 3 \mathrm{~K} 4 \mathrm{me} 3$ (Buard et al. 2009; Grey et al. 2011), we found here that H3K36me3 at hotspots is not a downstream consequence of DSB formation as it is detected in B6 Spo11 ${ }^{\text {KO }}$ mice. H3K36me3 may play a role in specifying DSB formation and/or in DSB repair, as described in somatic cells (Fnu et al. 2011; Carvalho et al. 2014; Pai et al. 2014; Pfister et al. 2014). In somatic cells, H3K36me3 favors DSB repair by homologous recombination through regulation of end processing (Clouaire and Legube 2015), and meiotic DSB repair is specifically channeled toward the homologous recombination pathway (Hunter 2015).

PRDM9 interacts with a subset of its genomic targets independently of its zinc finger specificity

In class 2 sites, the absence of detectable motifs that share similarity with PRDM9 consensus sequences strongly suggests that the PRDM9 zinc finger array does not interact with DNA, at least not in the canonical way. As these sites do not show the expected features of PRDM9 binding, it was important to exclude the possibility that they may result from technical artefacts. This was done by removing from our analysis peaks that were present in $\mathrm{B} 6 \mathrm{Prdm} 9^{K O}$, as well as peaks common to $\mathrm{B} 6$ and $\mathrm{RJ} 2$, which could potentially be false positives. The presence of some false-positive peaks, corresponding to recognition of another protein by our antiPRDM9 antibody, among those selected cannot be formally excluded. This would, however, imply the rather unlikely possibility that their detection is reproducible in the same genotype but not between the two strains. Furthermore, a large fraction of these sites (class 2B) do not overlap with regions of accessible chromatin that were considered as artifacts in some ChIP-seq analyses (Jain et al. 2015). Conversely, class $2 \mathrm{~A}$ sites, which mainly overlap with promoters and thus with accessible chromatin, were not detected systematically in all experiments as they were absent in B6 Spo11 ${ }^{K O}$.

Interestingly, PRDM9 class 2 sites differ in the RJ2 and B6 strains but contain no identified PRDM9 allele-specific binding motif. Therefore, we hypothesize that their location is somehow indirectly specified by the binding of PRDM9 with class 1 sites that is determined by the PRDM9 zinc finger array. For instance, proximity with a class 1 site could be a necessary feature for class 2 site interaction with PRDM9, and this interaction might, consequently, involve motif-bound PRDM9 rather than soluble PRDM9 (Fig. 6). We tested this hypothesis; however, we did not detect specific proximity (in base pairs) between class 1 (or DMC1 peaks) and class 2 sites. Therefore, additional factors could contribute to the selection of class 2 sites by PRDM9 bound to class 1 sites such as the three-dimensional chromatin organization. Whatever the mode of PRDM9 recruitment to these sites, we can conclude that PRDM9 interaction with these class 2 sites does not provide a proper context for DSB formation as no recombination could be detected at these sites.

\section{PRDM9 is recruited to promoters in a Spoll-dependent manner}

Class $2 \mathrm{~A}$ sites are mainly promoters that are mostly active at the beginning of meiotic prophase (99\% are expressed in spermatocytes at leptotene/zygotene) (da Cruz et al. 2016). Their Spo11 dependency could imply that DSB formation or progression throughout meiotic prophase is required for PRDM9 interaction with these sites. DSBs are induced within and around PRDM9 binding sites (Lange et al. 2016), and this could result in Spo11-dependent PRDM9 displacement. We suggest that this displacement leads to PRDM9 interaction with promoters. As mentioned above, we propose that the location of the promoter with which PRDM9 interacts depends on the location of the DSB sites. The molecular nature of this dependency remains to be determined (Fig. 6). It would also be interesting to determine whether PRDM9 is recruited to class $2 \mathrm{~A}$ sites through interaction with some transcription 
machinery components at these promoters. Whatever the mechanism leading to these alternative interactions, it also raises the question of their function. They could be a "by-product" of PRDM9 activity as a member of the PRDM family of transcription regulators (Fog et al. 2012), but they might also have a specific, not yet determined function.

\section{PRDM9 sites compatible with loop-axis interactions}

Class 2B sites combine the unexpected properties of PRDM9 binding in a DSB-independent manner without showing the expected consequences of this binding, namely, H3K4me3 and recombination, based on the DMC1 and GC* analyses. The mechanism of PRDM9 interaction with class $2 \mathrm{~B}$ sites remains to be investigated. CTCF could be involved in PRDM9 interaction with some class 2B sites. We detected the CTCF binding motif in $60 \%$ of class $2 \mathrm{~B}$ sites in the strain expressing PRDM9 ${ }^{\text {Dom2 }}$. In our coimmunoprecipitation assays, the PRDM9-CTCF interaction is sensitive to benzonase (Supplemental Fig. S6C). Therefore, it is more likely that this interaction is indirect and involves DNA or RNA. CTCF is an insulator protein that binds to promoters and enhancers, at the border of topologically associated domains (TADs), and its binding sites partially overlap with cohesin binding sites (Ong and Corces 2014). Given the cohesin enrichment along meiotic chromosome axes at the stage of DSB formation (McNicoll et al. 2013), we speculate that a fraction of CTCF could be axis-associated, as reported in somatic cells (Tedeschi et al. 2013). PRDM9 could thus interact indirectly with DNA sequences located on the axis, and the allele specificity of these sites favors a model where PRDM9 molecules bound to class 1 sites are involved in these interactions, as discussed above. An example of such indirect interactions involves the insulator protein BEAF-32 (Liang et al. 2014). According to this model, the class $2 \mathrm{~B}$ signal uncovered by our analysis reveals an interaction between two genomic loci: one DSB site and one axis-associated region (Fig. 6). Other genomic sites that do not include CTCF motifs are recovered through these proposed indirect interactions, particularly in the presence of PRDM9 ${ }^{\text {Cst }}$. It will be interesting to test whether they are associated with other axis proteins. In the RJ2 strain where the number of sites was the greatest, we could detect a strong correlation between the distribution of class $2 \mathrm{~B}$ and class 1 sites over 5- to $10-\mathrm{Mbp}$ intervals. This correlation is compatible with a loopaxis interaction involved in PRDM9 binding to these sites, which will lead to spatial constraints between class 1 and $2 \mathrm{~B}$ sites (Supplemental Fig. S4D). The evidence for loop-axis interaction in S. cerevisiae and its role on DSB formation was based on the mapping of axis and DSB sites, and the observation that several proteins essential for DSB formation (Rec114, Mei4, and Mer2, the RMM complex) and DSB repair are located on the chromosome axis. These RMM proteins, as well as axis proteins important for their localization (Hop1 and Red1), are not evenly distributed along chromosomes leading to domains with high or low meiotic DSB activity (Blat et al. 2002; Panizza et al. 2011). It is interesting to note that PRDM9 peak density is not evenly distributed along mouse chromosomes, and it will be interesting to evaluate whether it is related to features of chromosome organization. Two proteins required for meiotic DSB formation in mice (MEI4 and IHO1) are also located on the chromosomes axis (Kumar et al. 2010, 2015; Stanzione et al. 2016) and may also be unevenly distributed along chromosomes.

In conclusion, by monitoring PRDM9 binding in vivo, we provide unprecedented insights into the molecular interactions as- sociated with meiotic recombination hotspot activity. Our approach highlights the negative effect of hotspot erosion on PRDM9 affinity for DNA. The important level of hotspot erosion in the $M . m$. domesticus $\mathrm{C} 57 \mathrm{BL} / 6$ strains suggests that the $\operatorname{Prdm} 9^{\text {Dom } 2}$ allele has been active for many generations in this subspecies. As several steps are needed from PRDM9 binding to DSB formation, it is not surprising that the two events are only partially correlated. This brings interesting questions concerning the additional elements involved, which could include interactions with axis proteins that are predicted to be required for activation of the SPO11/TOPOVIBL complex. Our study provides the first analysis in the mouse suggesting that DSB sites interact with other, possibly axis-associated, genomic regions. Remarkably, we also discovered a new category of PRDM9 binding sites, where PRDM9 binding is Spo11-dependent. This finding outlines another challenge concerning the analysis of PRDM9 interaction dynamics during DSB formation and repair.

\section{Methods}

\section{Mouse strains}

The following mouse strains were used: C57BL/6JOlaHsd (B6), B10.MOLSGR(A)-(D17Mit58-D17Jcs11)/Bdm (RJ2) (Grey et al. 2009), B6;129P2-Prdm9 $9^{\text {tm1Ymat }} / J$ (B6 Prdm9 ${ }^{K O}$ ) (Hayashi et al. 2005), and $S p o 11^{t m 1 M}$. This $S p o 11^{K O / K O}$ strain (hereafter B6 $S p o 11^{K O}$ ) carries the Prdm $9^{\text {Dom } 2}$ allele from the B6 strain (Baudat et al. 2000). RJ2 has a C57BL/10 genetic background, which is highly similar to B6, and expresses $\operatorname{Prdm} 9^{\mathrm{Cst}}$ (previously named $\operatorname{Prdm} 9^{w m 7}$ ). B6 and B6 $S p o 11^{K O}$ express $\operatorname{Prdm} 9^{\operatorname{Dom} 2}$ (previously named $\operatorname{Prdm} 9^{b}$ ). All animal experiments were carried out according to the CNRS guidelines and approved by the ethics committee on live animals (project CE-LR-0812 and 1295).

\section{Antibodies}

Rabbit polyclonal antibodies against PRDM9 were developed as described in the Supplemental Methods. A list of the other antibodies used in this article is in the Supplemental Methods.

\section{ChIP-seq experiments and analysis}

For H3K4me3 and DMC1 ChIP-seq experiments, we used the protocols described by Buard et al. (2009) and Khil et al. (2012), respectively.

Details on PRDM9 and H3K36me3 ChIP-seq experiments are described in the Supplemental Methods. Numbers of uniquely mapped reads are shown in Supplemental Table S1.

\section{Computational data analysis}

For ChIP-seq data processing, reads were mapped to the UCSC build mm9 mouse genome assembly. Peaks were identified from unique and high-quality mapped reads. Detailed procedure and parameters of peak calling and downstream analysis are given in the Supplemental Methods.

\section{GC-biased gene conversion signature analysis}

Substitutions that occurred in the M. m. castaneus lineage or in the M. $m$. domesticus lineage were identified by comparison with the Mus spretus outgroup. Details are given in the Supplemental Methods.

\section{Genome Research}

www.genome.org 


\section{Immunostaining}

Immunostaining experiments using histological sections and chromosome spreads are detailed in the Supplemental Methods.

\section{PRDM9 protein extraction, immunoprecipitation, and Western blotting}

Nuclear extracts were prepared as described earlier (Dignam et al. 1983). Details for immunoprecipitation and Western blot procedures are described in the Supplemental Methods.

\section{Data access}

The raw and processed sequencing files produced in this study (ChIP-seq data listed in Supplemental Table S1) have been submitted to the NCBI Gene Expression Omnibus (GEO; http://www.ncbi .nlm.nih.gov/geo/) under accession number GSE93955.

\section{Acknowledgments}

We thank all laboratory members for discussion and specifically Frédéric Baudat for comments, suggestions, and insight during the whole project and Christine Brun for cytological analysis. We thank Claire Guillet for performing the initial analysis of H3K4me3 ChIP-seq data and Boyan Bonev and Jia-Ming Chan for discussions. We thank Jacques van Helden for suggestions for motif analysis and F. Pratto and K. Brick for information and help in setting up the DMC1 ChIP-seq experiments and analysis. Sequencing and microscopy analyses were performed at the MGX (Montpellier GenomiX) and MRI (Montpellier Ressources Imagerie) facilities, respectively. We thank RAM (Réseau des animaleries de Montpellier) and specially Frederic Gallardo from the mouse facility. The work leading to the H3K4me3 results received funding from the European Union Seventh Framework Programme (FP7/2007-2013) under grant agreement no. 262055. This work was supported by the Agence Nationale de la Recherche (ANR-15-CE12-0010-01/DaSiRe). B.d.M. was funded by grants from the Centre National pour la Recherche Scientifique (CNRS) and the European Research Council Executive Agency under the European Community's Seventh Framework Programme (FP7/2007-2013 grant agreement no. [322788]).

Author contributions: B.d.M. and C.G. conceived the project. C.G. performed the experiments. J.A.J.C. performed the bioinformatic analysis. J.B. performed the H3K4me3 experiments, analyzed by B.L. I.G. and M.G. provided the H3K4me3 sequencing data. L.D. analyzed the GC content. B.d.M. and C.G. wrote the manuscript. B.d.M., C.G., J.A.J.C., and L.D. revised the manuscript.

\section{References}

Acquaviva L, Szekvolgyi L, Dichtl B, Dichtl BS, de La Roche Saint Andre C, Nicolas A, Geli V. 2013. The COMPASS subunit Spp1 links histone methylation to initiation of meiotic recombination. Science 339: 215-218.

Auton A, Rui Li Y, Kidd J, Oliveira K, Nadel J, Holloway JK, Hayward JJ, Cohen PE, Greally JM, Wang J, et al. 2013. Genetic recombination is targeted towards gene promoter regions in dogs. PLoS Genet 9: e1003984.

Axelsson E, Webster MT, Ratnakumar A, Ponting CP, Lindblad-Toh K. 2012. Death of PRDM9 coincides with stabilization of the recombination landscape in the dog genome. Genome Res 22: 51-63.

Aymard F, Bugler B, Schmidt CK, Guillou E, Caron P, Briois S, Iacovoni JS, Daburon V, Miller KM, Jackson SP, et al. 2014. Transcriptionally active chromatin recruits homologous recombination at DNA double-strand breaks. Nat Struct Mol Biol 21: 366-374.
Baker CL, Walker M, Kajita S, Petkov PM, Paigen K. 2014. PRDM9 binding organizes hotspot nucleosomes and limits Holliday junction migration. Genome Res 24: 724-732.

Baker CL, Kajita S, Walker M, Saxl RL, Raghupathy N, Choi K, Petkov PM, Paigen K. 2015. PRDM9 drives evolutionary erosion of hotspots in Mus musculus through haplotype-specific initiation of meiotic recombination. PLoS Genet 11: e1004916.

Baudat F, Manova K, Yuen JP, Jasin M, Keeney S. 2000. Chromosome synapsis defects and sexually dimorphic meiotic progression in mice lacking spo11. Mol Cell 6: 989-998.

Baudat F, Buard J, Grey C, Fledel-Alon A, Ober C, Przeworski M, Coop G, de Massy B. 2010. PRDM9 is a major determinant of meiotic recombination hotspots in humans and mice. Science 327: 836-840.

Baudat F, Imai Y, de Massy B. 2013. Meiotic recombination in mammals: localization and regulation. Nat Rev Genet 14: 794-806.

Berg IL, Neumann R, Lam KW, Sarbajna S, Odenthal-Hesse L, May CA, Jeffreys AJ. 2010. PRDM9 variation strongly influences recombination hot-spot activity and meiotic instability in humans. Nat Genet 42: 859-863.

Berg IL, Neumann R, Sarbajna S, Odenthal-Hesse L, Butler NJ, Jeffreys AJ. 2011. Variants of the protein PRDM9 differentially regulate a set of human meiotic recombination hotspots highly active in African populations. Proc Natl Acad Sci 108: 12378-12383.

Blat Y, Protacio RU, Hunter N, Kleckner N. 2002. Physical and functional interactions among basic chromosome organizational features govern early steps of meiotic chiasma formation. Cell 111: 791-802.

Borde V, de Massy B. 2013. Programmed induction of DNA double strand breaks during meiosis: setting up communication between DNA and the chromosome structure. Curr Opin Genet Dev 23: 147-155.

Borde V, Robine N, Lin W, Bonfils S, Geli V, Nicolas A. 2009. Histone H3 lysine 4 trimethylation marks meiotic recombination initiation sites. EMBO J 28: 99-111.

Brick K, Smagulova F, Khil P, Camerini-Otero RD, Petukhova GV. 2012. Genetic recombination is directed away from functional genomic elements in mice. Nature 485: 642-645.

Buard J, Barthes P, Grey C, de Massy B. 2009. Distinct histone modifications define initiation and repair of meiotic recombination in the mouse. EMBO J 28: 2616-2624.

Buard J, Rivals E, Dunoyer de Segonzac D, Garres C, Caminade P, de Massy B, Boursot P. 2014. Diversity of Prdm9 zinc finger array in wild mice unravels new facets of the evolutionary turnover of this coding minisatellite. PLoS One 9: e85021.

Carvalho S, Vitor AC, Sridhara SC, Martins FB, Raposo AC, Desterro JM, Ferreira J, de Almeida SF. 2014. SETD2 is required for DNA double-strand break repair and activation of the p53-mediated checkpoint. eLife 3: e02482.

Chen JM, Cooper DN, Chuzhanova N, Ferec C, Patrinos GP. 2007. Gene conversion: mechanisms, evolution and human disease. Nat Rev Genet 8: $762-775$.

Clement Y, Arndt PF. 2013. Meiotic recombination strongly influences GCcontent evolution in short regions in the mouse genome. Mol Biol Evol 30: $2612-2618$.

Clouaire T, Legube G. 2015. DNA double strand break repair pathway choice: a chromatin based decision? Nucleus 6: 107-113.

Coop G, Przeworski M. 2007. An evolutionary view of human recombination. Nat Rev Genet 8: 23-34.

da Cruz I, Rodriguez-Casuriaga R, Santinaque FF, Farias J, Curti G, Capoano CA, Folle GA, Benavente R, Sotelo-Silveira JR, Geisinger A. 2016. Transcriptome analysis of highly purified mouse spermatogenic cell populations: Gene expression signatures switch from meiotic-to postmeiotic-related processes at pachytene stage. BMC Genomics 17: 294.

Davies B, Hatton E, Altemose N, Hussin JG, Pratto F, Zhang G, Hinch AG, Moralli D, Biggs D, Diaz R, et al. 2016. Re-engineering the zinc fingers of PRDM9 reverses hybrid sterility in mice. Nature 530: 171-176.

de Massy B. 2013. Initiation of meiotic recombination: how and where? Conservation and specificities among eukaryotes. Annu Rev Genet 47: 563-599.

Dignam JD, Martin PL, Shastry BS, Roeder RG. 1983. Eukaryotic gene transcription with purified components. Methods Enzymol 101: 582-598.

Duret L, Galtier N. 2009. Biased gene conversion and the evolution of mammalian genomic landscapes. Annu Rev Genomics Hum Genet 10: 285-311.

Eram MS, Bustos SP, Lima-Fernandes E, Siarheyeva A, Senisterra G, Hajian T, Chau I, Duan S, Wu H, Dombrovski L, et al. 2014. Trimethylation of histone H3 lysine 36 by human methyltransferase PRDM9 protein. J Biol Chem 289: 12177-12188.

Fnu S, Williamson EA, De Haro LP, Brenneman M, Wray J, Shaheen M, Radhakrishnan K, Lee SH, Nickoloff JA, Hromas R. 2011. Methylation of histone H3 lysine 36 enhances DNA repair by nonhomologous end-joining. Proc Natl Acad Sci 108: 540-545. 
Fog CK, Galli GG, Lund AH. 2012. PRDM proteins: important players in differentiation and disease. Bioessays 34: 50-60.

Grey C, Baudat F, de Massy B. 2009. Genome-wide control of the distribution of meiotic recombination. PLoS Biol 7: e35.

Grey C, Barthes P, Chauveau-Le Friec G, Langa F, Baudat F, de Massy B. 2011 Mouse PRDM9 DNA-binding specificity determines sites of histone $\mathrm{H} 3$ lysine 4 trimethylation for initiation of meiotic recombination. PLoS Biol 9: e1001176.

Hammoud SS, Low DH, Yi C, Carrell DT, Guccione E, Cairns BR. 2014. Chromatin and transcription transitions of mammalian adult germline stem cells and spermatogenesis. Cell Stem Cell 15: 239-253.

Hayashi K, Yoshida K, Matsui Y. 2005. A histone H3 methyltransferase controls epigenetic events required for meiotic prophase. Nature 438 $374-378$.

Hunter N. 2015. Meiotic recombination: the essence of heredity. Cold Spring Harb Perspect Biol 7: a016618.

Jain D, Baldi S, Zabel A, Straub T, Becker PB. 2015. Active promoters give rise to false positive 'Phantom Peaks' in ChIP-seq experiments. Nucleic Acids Res 43: 6959-6968.

Jeffreys AJ, Cotton VE, Neumann R, Lam KW. 2013. Recombination regulator PRDM9 influences the instability of its own coding sequence in humans. Proc Natl Acad Sci 110: 600-605.

Khil PP, Smagulova F, Brick KM, Camerini-Otero RD, Petukhova GV. 2012. Sensitive mapping of recombination hotspots using sequencing-based detection of ssDNA. Genome Res 22: 957-965.

Koh-Stenta X, Joy J, Poulsen A, Li R, Tan Y, Shim Y, Min JH, Wu L, Ngo A, Peng J, et al. 2014. Characterization of the histone methyltransferase PRDM9 using biochemical, biophysical and chemical biology techniques. Biochem J 461: 323-334.

Kono H, Tamura M, Osada N, Suzuki H, Abe K, Moriwaki K, Ohta K, Shiroishi T. 2014. Prdm9 polymorphism unveils mouse evolutionary tracks. DNA Res 21: 315-326.

Kumar R, Bourbon HM, de Massy B. 2010. Functional conservation of Mei4 for meiotic DNA double-strand break formation from yeasts to mice. Genes Dev 24: 1266-1280.

Kumar R, Ghyselinck N, Ishiguro K, Watanabe Y, Kouznetsova A, Hoog C, Strong E, Schimenti J, Daniel K, Toth A, et al. 2015. MEI4: a central player in the regulation of meiotic DNA double-strand break formation in the mouse. J Cell Sci 128: 1800-1811.

Lange J, Yamada S, Tischfield SE, Pan J, Kim S, Zhu X, Socci ND, Jasin M, Keeney S. 2016. The landscape of mouse meiotic double-strand break formation, processing, and repair. Cell 167: 695-708.e616.

Liang J, Lacroix L, Gamot A, Cuddapah S, Queille S, Lhoumaud P, Lepetit P, Martin PG, Vogelmann J, Court F, et al. 2014. Chromatin immunoprecipitation indirect peaks highlight long-range interactions of insulator proteins and Pol II pausing. Mol Cell 53: 672-681.

McNicoll F, Stevense M, Jessberger R. 2013. Cohesin in gametogenesis. Curr Topics Dev Biol 102: 1-34.

Munoz-Fuentes V, Di Rienzo A, Vila C. 2011. Prdm9, a major determinant of meiotic recombination hotspots, is not functional in dogs and their wild relatives, wolves and coyotes. PLoS One 6: e25498.

Myers S, Bowden R, Tumian A, Bontrop RE, Freeman C, MacFie TS, McVean G, Donnelly P. 2010. Drive against hotspot motifs in primates implicates the PRDM9 gene in meiotic recombination. Science 327: 876-879.

Nakahashi H, Kwon KR, Resch W, Vian L, Dose M, Stavreva D, Hakim O, Pruett N, Nelson S, Yamane A, et al. 2013. A genome-wide map of CTCF multivalency redefines the CTCF code. Cell Rep 3: 1678-1689.

Ong CT, Corces VG. 2014. CTCF: an architectural protein bridging genome topology and function. Nat Rev Genet 15: 234-246.

Pai CC, Deegan RS, Subramanian L, Gal C, Sarkar S, Blaikley EJ, Walker C Hulme L, Bernhard E, Codlin S, et al. 2014. A histone H3K36 chromatin switch coordinates DNA double-strand break repair pathway choice. Nat Commun 5: 4091

Pan J, Sasaki M, Kniewel R, Murakami H, Blitzblau HG, Tischfield SE, Zhu X, Neale MJ, Jasin M, Socci ND, et al. 2011. A hierarchical combination of factors shapes the genome-wide topography of yeast meiotic recombination initiation. Cell 144: 719-731.
Panizza S, Mendoza MA, Berlinger M, Huang L, Nicolas A, Shirahige K, Klein F. 2011. Spo11-accessory proteins link double-strand break sites to the chromosome axis in early meiotic recombination. Cell 146: $372-383$.

Parvanov ED, Petkov PM, Paigen K. 2010. Prdm9 controls activation of mammalian recombination hotspots. Science 327: 835

Pfister SX, Ahrabi S, Zalmas LP, Sarkar S, Aymard F, Bachrati CZ, Helleday T, Legube G, La Thangue NB, Porter AC, et al. 2014. SETD2-dependent histone H3K36 trimethylation is required for homologous recombination repair and genome stability. Cell Rep 7: 2006-2018.

Powers NR, Parvanov ED, Baker CL, Walker M, Petkov PM, Paigen K. 2016. The meiotic recombination activator PRDM9 trimethylates both H3K36 and H3K4 at recombination hotspots in vivo. PLoS Genet 12: e1006146.

Pratto F, Brick K, Khil P, Smagulova F, Petukhova GV, Camerini-Otero RD. 2014. DNA recombination. Recombination initiation maps of individual human genomes. Science 346: 1256442.

Pugacheva EM, Rivero-Hinojosa S, Espinoza CA, Mendez-Catala CF, Kang S, Suzuki T, Kosaka-Suzuki N, Robinson S, Nagarajan V, Ye Z, et al. 2015. Comparative analyses of CTCF and BORIS occupancies uncover two distinct classes of CTCF binding genomic regions. Genome Biol 16: 161.

Robert T, Nore A, Brun C, Maffre C, Crimi B, Guichard V, Bourbon HM, de Massy B. 2016. The TopoVIB-Like protein family is required for meiotic DNA double-strand break formation. Science 351: 943-949.

Romanienko PJ, Camerini-Otero RD. 2000. The mouse Spo11 gene is required for meiotic chromosome synapsis. Mol Cell 6: 975-987.

Singhal S, Leffler EM, Sannareddy K, Turner I, Venn O, Hooper DM, Strand AI, Li Q, Raney B, Balakrishnan CN, et al. 2015. Stable recombination hotspots in birds. Science 350: 928-932.

Smagulova F, Gregoretti IV, Brick K, Khil P, Camerini-Otero RD, Petukhova GV. 2011. Genome-wide analysis reveals novel molecular features of mouse recombination hotspots. Nature 472: 375-378.

Smagulova F, Brick K, Pu Y, Camerini-Otero RD, Petukhova GV. 2016. The evolutionary turnover of recombination hot spots contributes to speciation in mice. Genes Dev 30: 266-280.

Sommermeyer V, Beneut C, Chaplais E, Serrentino ME, Borde V. 2013. Spp1, a member of the Set1 Complex, promotes meiotic DSB formation in promoters by tethering histone H3K4 methylation sites to chromosome axes. Mol Cell 49: 43-54.

Stanzione M, Baumann M, Papanikos F, Dereli I, Lange J, Ramlal A, Trankner D, Shibyua H, de Massy B, Watanabe Y, et al. 2016. Meiotic DNA break formation requires the unsynapsed chromosome axis-binding protein IHO1 (CCDC36) in mice. Nat Cell Biol 18: 1208-1220.

Tan M, Luo H, Lee S, Jin F, Yang JS, Montellier E, Buchou T, Cheng Z, Rousseaux S, Rajagopal N, et al. 2011. Identification of 67 histone marks and histone lysine crotonylation as a new type of histone modification. Cell 146: $1016-1028$.

Tedeschi A, Wutz G, Huet S, Jaritz M, Wuensche A, Schirghuber E, Davidson IF, Tang W, Cisneros DA, Bhaskara V, et al. 2013. Wapl is an essential regulator of chromatin structure and chromosome segregation. Nature 501: $564-568$.

Thacker D, Mohibullah N, Zhu X, Keeney S. 2014. Homologue engagement controls meiotic DNA break number and distribution. Nature 510: 241-246.

Vrielynck N, Chambon A, Vezon D, Pereira L, Chelysheva L, De Muyt A, Mezard C, Mayer C, Grelon M. 2016. A DNA topoisomerase VI-like complex initiates meiotic recombination. Science 351: 939-943.

Wu H, Mathioudakis N, Diagouraga B, Dong A, Dombrovski L, Baudat F, Cusack S, de Massy B, Kadlec J. 2013. Molecular basis for the regulation of the H3K4 methyltransferase activity of PRDM9. Cell Rep 5: 13-20.

Yue F, Cheng Y, Breschi A, Vierstra J, Wu W, Ryba T, Sandstrom R, Ma Z, Davis C, Pope BD, et al. 2014. A comparative encyclopedia of DNA elements in the mouse genome. Nature 515: 355-364.

Zickler D, Kleckner N. 1999. Meiotic chromosomes: integrating structure and function. Annu Rev Genet 33: 603-754.

Received October 18, 2016; accepted in revised form February 23, 2017. 


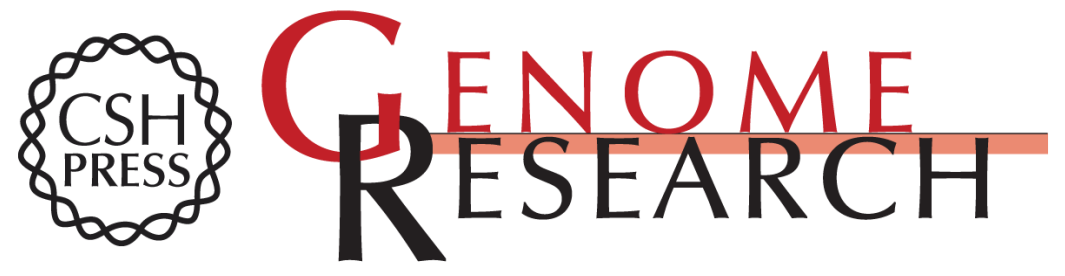

\section{In vivo binding of PRDM9 reveals interactions with noncanonical genomic sites}

Corinne Grey, Julie A.J. Clément, Jérôme Buard, et al.

Genome Res. 2017 27: 580-590 originally published online March 23, 2017

Access the most recent version at doi:10.1101/gr.217240.116

\section{Supplemental} Material

References

Open Access

Creative Commons License

Email Alerting Service
http://genome.cshlp.org/content/suppl/2017/03/23/gr.217240.116.DC1

This article cites 71 articles, 22 of which can be accessed free at: http://genome.cshlp.org/content/27/4/580.full.html\#ref-list-1

Freely available online through the Genome Research Open Access option.

This article, published in Genome Research, is available under a Creative Commons License (Attribution 4.0 International), as described at http://creativecommons.org/licenses/by/4.0/.

Receive free email alerts when new articles cite this article - sign up in the box at the top right corner of the article or click here.

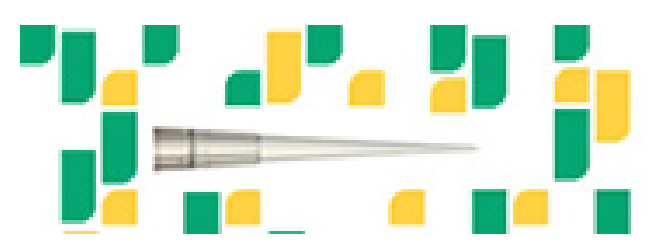

Focused on your science.

\section{Jכז}

SCIENTIFIC

suob or seisnes

To subscribe to Genome Research go to: https://genome.cshlp.org/subscriptions 